\title{
LA FORMACIÓN INICIAL EN EDUCACIÓN FÍSICA. NOTAS PARA PROBLEMATIZAR LA RELACIÓN ENTRE TEORÍA Y PRÁCTICA EN LA FORMACIÓN TERCIARIA NO UNIVERSITARIA
}

\author{
Norma Beatriz Rodríguez Feilberg 1 \\ María Jimena Galmez²
}

\begin{abstract}
RESUMEN
Este artículo analiza las vinculaciones entre teoría y práctica en la Formación de Profesores/as en Educación Física. La investigación se realizó en el Instituto Superior de Formación Docente $N^{\circ} 84$ de la ciudad de Mar del Plata, provincia de Buenos Aires, Argentina, durante el ciclo lectivo 2020. La hipótesis de trabajo es que la reconversión de la enseñanza mediada por herramientas de educación a distancia -producto de la pandemia por COVID 19- expuso a la Educación Física como un área de conocimiento cuyos límites se delimitan en la práctica. El estudio implicó un análisis documental y entrevistas a profesores y profesoras en Educación Física que se desempeñan como docentes de la institución. Los resultados muestran que en la formación conviven diversas perspectivas teóricas y que los modos de afrontar la particular coyuntura, producto de la emergencia sanitaria, fueron variados y heterogéneos.
\end{abstract}

Palavras-chave: Formación superior. Teoría. Práctica. Educación Física.

\section{TREINAMENTO INICIAL EM EDUCAÇÃO FÍSICA. NOTAS PARA PROBLEMATIZAR A RELAÇÃO ENTRE TEORIA E PRÁTICA NA FORMAÇÃO SUPERIOR NÃO UNIVERSITÁRIA}

\footnotetext{
${ }^{1}$ Magíster en Educación Corporal. Universidad Nacional de la Plata. (UNLP) y Profesora en Educación Física (UNLP). Se desempeña como Profesora Titular en la Teoría de la Educación Física 4. DEF-FaHCE-UNLP. Miembro del Comité Asesor de CICES (Centro de Investigación Cuerpo, Educación y Sociedad) perteneciente al CICES - IdIHCS/ FaHCE/UNLP - CONICET. Investigadora Categoría III del Programa de incentivos a la investigación. Directora del Proyecto Producción Científica y Formación de Educadores Corporales. Estudio sobre la Formación inicial de profesores en Educación Física en la UNLP / H951. (Proyecto de investigación). UNLP. FaHCE. E-mail:nbrodri@gmail.com. Sitio oficial de la UNLP: http://www.memoria.fahce.unlp.edu.ar/perfiles/1173RodriguezN Orcid iD: https://orcid.org/0000-0003-0250-1477. E-mail: nbrodri@gmail.com

${ }^{2}$ Universidad Nacional de La Plata- Facultad de Humanidades y Ciencias de la EducaciónArgentina. Profesora de Educación Física (ISFDT N 81). Estudiante de la Licenciatura en Educación Física (UNLP). Investigadora en Formación del CICES - IdlHCS/ FaHCE/UNLP CONICET. Orcid iD: https://orcid.org/0000-0003-3041-0927. E-mail: mjgalmez@gmial.com
} 


\title{
RESUMO
}

Este artigo analisa as ligações entre teoria e prática na Formação de Professores de Educação Física. A pesquisa foi realizada no Instituto Superior de Formação de Professores $n^{\circ} 84$ da cidade de Mar del Plata, província de Buenos Aires, Argentina, durante o ano letivo de 2020. A hipótese de trabalho é que a reconversão do ensino mediada por ferramentas educacionais à distância produto da pandemia COVID 19 expôs a Educação Física como uma área do conhecimento cujos limites são delimitados na prática. O estudo envolveu análise documental e entrevistas com professores de Educação Física que atuam como docentes da instituição. Os resultados mostram que diversas perspectivas teóricas coexistem na formação e que as formas de enfrentamento à situação particular decorrente da emergência sanitária foram variadas e heterogêneas.

Palavras-chave: Ensino superior. Teoria. Prática. Educação Física.

\section{PE TEACHER TRAINING. NOTES TO PROBLEMATIZE THE RELATIONSHIP BETWEEN THEORY AND PRACTICE IN NON-UNIVERSITY EDUCATION}

\begin{abstract}
In this paper we analyze the links between theory and practice in Physical Education Teachers' training. The research was developed in the "Instituto Superior de Formación Docente 84" in Mar del Plata, Buenos Aires, Argentina, in 2020. Our working hypothesis is that the reorganization and transformation of teaching by means of remote education tools due to the COVID 19 pandemic exposed Physical Education as an area of knowledge whose limits are delimited in practice. The study involved a documentary analysis and interviews with professors in Physical Education at the aforementioned institution. The results show that various theoretical perspectives coexist in the education of Physical Education Teachers and that diverse and heterogeneous approaches were used to face the particular situation resulting from the health emergency.
\end{abstract}

Keywords: Teacher Education. Theory. Practice. Physical Education.

\section{INTRODUCCIÓN}

Esta investigación es el resultado de una de las líneas de pesquisa que se desprende del proyecto Producción Científica y Formación de Educadores Corporales. Estudio sobre la Formación inicial de profesores en Educación Física en la UNLP / H951 evaluado y financiado por la Secretaría de Políticas Universitarias, dependiente del Ministerio de Educación de la República Argentina. Se trata de un proyecto de cuatro años en el que interviene un equipo de investigación y cuyo propósito es "profundizar el 
concepto de saber con el que opera la enseñanza de la Educación Física en la Formación Superior3".

Es sabido que los estudios sobre Formación Superior en Argentina son frecuentes y sus complejidades múltiples. La formación de profesores/as se realiza, por un lado, en Universidades con opciones de gestión pública o privada. En este último caso, los planes de estudio se encuentran elaborados por cada Universidad de manera independiente. A su vez, existen Institutos de Formación Docente (no Universitaria) que dependen de las Direcciones de Educación de cada provincia. Aquí, dicha entidad es la responsable de elaborar el diseño curricular. En este trabajo se presenta el estudio de caso realizado en el Instituto Superior de Formación Docente $N^{0}$ 84, un establecimiento no- universitario, de gestión estatal y pública, ubicado en la ciudad de Mar del Plata, provincia de Buenos Aires, Argentina, durante el ciclo lectivo 2020. Esta investigación implicó una revisión del diseño curricular, un análisis de la situación del ASPO 2020 y la implementación y análisis de entrevistas a profesores y profesoras de la institución.

En primer lugar, se presentan las perspectivas de currículo que se seleccionaron. Siendo así, el currículo, un concepto que abarca pluralidades de significados, habiendo sido objeto de innumerables estudios y diversos enfoques. "El currículum es el documento oficial -o el conjunto de documentos- que materializa el proceso de selección y traducción cultural que origina el contenido a enseñar." (GVIRTZ Y PALAMIDESSI; 1998; p. 16).

Se trata de un término que ha permitido, tras su análisis contextual, abordar la realidad educativa. Concepto que, a su vez, posee una función más técnica al constituirse como herramienta de regulación pedagógica. Así, como documento oficial, el currículo es un lugar en donde se plasma una política educativa. Alicia de Alba precisa aún más esta cuestión:

Se entiende a la síntesis de elementos culturales: (conocimientos, valores, creencias, costumbres, hábitos) que conforman una propuesta político-educativa pensada e impulsada por diversos grupos y sectores sociales cuyos intereses son diversos y

3 http://www.memoria.fahce.unlp.edu.ar/library?a=d\&c=proyecto\&d=Jpy995 
contradictorios, aunque algunos tienden a ser dominantes y hegemónicos, y otros tiendan a oponerse y resistirse a tal dominación o hegemonía (ALBA, A; 2007; p.3).

El currículum es el principal mecanismo de la distribución del saber, ya que en él se determina qué contenidos se van a enseñar en cada nivel e institución escolar. Representa aspiraciones, metas, principios, en un contexto histórico concreto, afectados por las preferencias políticas, económicas y culturales, lo cual evidencia un contexto no neutral.

En este marco regulador se inscribe la educación escolar y es el Currículum un dispositivo específico siendo decisivo como matriz organizadora de contenidos. De esta manera, se suma a otras formas de regulación de los Estados emergentes como los de grupo-clase para distinguir a unos alumnos de otros y agruparlos en categorías que los definan y los clasifiquen.= (Vallina, J.; Rodríguez, N.; 2015; p. 1).

En el caso estudiado, el currículum es elaborado por la Dirección General de Cultura y Educación de la provincia de Buenos Aires y aprobado por RESOLUCIÓN No 2432. El diseño curricular de Formación Docente en Educación Física de la Provincia de Buenos Aires se posiciona en un paradigma articulador configurado por una serie de elementos que se interrelacionan con otras lógicas: "la lógica disciplinar, la lógica psicológica, la lógica de los saberes pedagógico-didácticos y la lógica de la práctica docente. Pero en él se privilegia la lógica articulatoria, producida por la perspectiva y el significado sociocultural." (DGCE, 2009: p. 26)

La formación de Profesores de Educación Física en el diseño curricular se organiza en cuatro años lectivos con un total de 3.296 horas. Las materias se estructuran en dos ciclos: formación básica común y la formación especializada en respuesta a lo que se expresa en la Ley de Educación Nacional (Ley 26206/06), categorizados a su vez en campos. Constituyen la formación básica común: el campo de la actualización formativa, el campo de la fundamentación y el campo el de la subjetividad y de las culturas. Mientras que, el ciclo de la formación especializada consta de: el campo de 
los saberes específicos, el campo articulador de la práctica docente y los talleres formativos opcionales (TFO). ${ }^{4}$

\section{REFERENTE TEÓRICO}

\section{Los saberes docentes}

Es indiscutible que las y los docentes se ubican en una posición imprescindible dentro del sistema educativo y los colectivos que constituyen la cultura intelectual y científica. Esta interrelación se concreta a partir de instituciones como las Universidades, organismos interesados por la producción y formación de conocimientos, en su misión de investigación y enseñanza. Pero, estos dos grupos (de investigación y enseñanza) se diferencian cada vez más. Los educadores se especializan en la transmisión y los investigadores en la producción sin ninguna relación entre sí. El saber del profesorado parece que existiera únicamente en la competencia técnica y pedagógica para transmitir saberes confeccionados por otros. El cuerpo docente es portador y transmisor de dichos saberes, realizando una selección, secuenciación y jerarquización de los contenidos de la enseñanza. Contenidos que, en general, son producidos por otros. En este caso, el docente, tiene una relación de alienación con el saber. Hay cierto distanciamiento (social, epistemológico, institucional) que lo separa y lo hace ajeno de esos saberes legitimados por otros. Se podría hablar de una relación exterior, donde se incorporan a la práctica docente sin que sean controlados, producidos o legitimados por ella.

En oposición a esta dimensión "técnica" del saber, podemos decir que los saberes y su producción constituyen dos polos complementarios e inseparables donde el cuerpo docente posee una importante función social y estratégica, equivalente a los grupos productores de saber. Ello, mientras que la función de los docentes no se reduzca a una mera reproducción, sino

4 Confrontar Diseño Curricular para la Formación Superior. DGCyE. http://servicios2.abc.gov.ar/lainstitucion/organismos/consejogeneral/disenioscurriculares/do cumentosdescarga/res2432-09fd_educ_fisica.pdf 
que su práctica no sólo integre, sino que también, inherentemente, produzca saberes.

Tardif, M, (2004) concibe al saber docente como saber plural, donde se integran y relacionan saberes provenientes de la formación profesional y disciplinares, curriculares, y experienciales, donde se envuelven en el propio ejercicio del trabajo conocimientos y un "saber hacer" diverso. A continuación, se describen dichos saberes:

Saber Profesional: son aquellos transmitidos por las instituciones de formación del profesorado. No se limita en la producción de conocimientos sino también, implica su incorporación a la práctica profesional. Estos saberes, provienen de reflexiones sobre la práctica educativa. En este caso, esta no es sólo un objeto de saber de las ciencias, sino que también es la actividad que moviliza diversos saberes.

Saberes Disciplinarios: son aquellos definidos y seleccionados por la institución, que nacen de la tradición cultural y de los grupos sociales productores de saberes.

Saberes Curriculares: son aquellos presentados y categorizados por la institución escolar y que responden a discursos, objetivos, contenidos y métodos.

Saberes Experienciales: devienen de los mismos maestros en el ejercicio de sus funciones, a partir de su práctica y el conocimiento de su medio. "Se incorporan a la experiencia individual y colectiva en forma de hábitos y de habilidades, de saber hacer y de saber ser. Podemos llamarlos "saberes experienciales o prácticos". (TARDIF, M; 2004 ;30)

Estos saberes se encuentran articulados e integrados en la práctica docente. Ahora bien, vale aquí preguntarnos, ¿̇cómo es esa relación?

\footnotetext{
Foucault entiende por "saber" las delimitaciones y las relaciones entre: 1) aquello de lo cual se puede hablar en una práctica discursiva (el dominio de los objetos); 2) el espacio donde el sujeto puede ubicarse para hablar de los objetos (posiciones subjetivas); 3) el campo de coordinación y de subordinación de los enunciados en los que los conceptos aparecen, que definen, se aplican y se transforman; 4) las posibilidades de utilización y de apropiación de los discursos (A S, 238) (CASTRO, E.; 2018: 364).
} 
El saber no es la suma de conocimientos, ni se reduce a un juicio verdadero respecto a algo (un hecho $u$ acción), tampoco a teorías con base empírica. Implica un dominio y también el ser capaz de determinar por qué razones es verdadero ese enunciado. Así se supera la lógica de saber cómo exclusivamente empírico, para considerarlo discursivo, en tanto hay sujeto.

\section{2 - Pesquisa sobre el documento curricular:}

En función de nuestros objetivos de diagnóstico/investigación, el trabajo de pesquisa implicó una búsqueda y análisis de los modos en los que la vinculación entre la teoría y la práctica se manifiestan en el diseño curricular. El estudio minucioso de dicho documento muestra que, por un lado, reitera tradiciones disciplinarias propias de la región y por el otro, este currículo postula que la Educación Física se inscribe en un paradigma interpretativo.

\footnotetext{
La investigación en la Formación Docente en Educación Física se inscribe en una tradición disciplinar, en las que se pueden visualizar posicionamientos epistemológicos hegemónicos ligados a la ciencia positivista. Esto ha supuesto, la separación entre la producción de teoría y la práctica, con la diferencial legitimación de sus productores y el aislamiento de las producciones de las distintas tramas culturales en el que las prácticas tenían lugar, así como la subordinación del campo de la Educación Física y el abandono de un saber constitutivo de la disciplina por el de disciplinas con mayor legitimación social.

La consideración de la Educación Física como una práctica social inscribe a la disciplina en el marco de las Ciencias Sociales, y en un posicionamiento epistemológico interpretativo (DCPBA: 2009:85).
}

Si bien, es probable que puedan rastrearse algunas muestras de los modos en los cuáles se constituyó el campo y en esa matriz fundante, las relaciones de subordinación, lo cierto es que, en nuestro país, no se escapa de lógicas epistemológicas regionales. El trabajo de Santiago Pich (2014), para el caso brasilero, presenta algunas perspectivas de interpretación. El en el texto: La formación de formadores y de profesores de Educación Física en Brasil: la tensión entre la producción de conocimiento y la identidad epistemológica, el autor sostiene que en los diseños curriculares de la 
formación superior de Brasil se observa una fuerte presencia de las "ciencias" y argumenta:

(...), lo que es fruto del proyecto cientificista de la Educación Física brasileña (Bracht, 2000), resultando en el modelo curricular denominado por Betti \& Betti (1996) currículum de orientación técnico-científica. En ese sentido, podemos decir que estamos ante la presencia de colectivos docentes de carácter heteróclito en los cuales se diferencian los (17) "teóricos-cientistas", responsables de las cátedras de los "fundamentos" tanto de las ciencias naturales como de las ciencias sociales, de los "prácticos" que permanecen concentrados en las cátedras de carácter didáctico-metodológico de las diferentes prácticas corporales tematizadas a lo largo de la formación (PICH, S.; 2014: 17- 18).

Esta división en campo de la formación y campo específico disciplinar es la misma que se observa en el diseño curricular que estudiamos. Así, en el campo de la formación se desarrollan temas y problemas ligados al conocimiento científico en el campo de las ciencias sociales y naturales y en el campo de la formación específica, se despliegan contenidos enmarcados en temas y problemas metodológicos de la didáctica y de las prácticas de intervención. Asimismo, se visualiza que, lo que trata de analizar el autor es de qué modo se manifiesta el colonialismo epistemológico en donde la escisión entre teoría y práctica es insalvable.

\begin{abstract}
Ahí se presentan dos problemas importantes: por un lado, los "teóricos" trabajan en la perspectiva de las "sub-áreas de conocimiento" (Bracht, 2000), orientando su trabajo de investigación y docencia por problemas y procedimientos metodológicos de las ciencias de origen, a lo que Gamboa (2007) llamó "colonialismo epistemológico". Además, esos actores no consiguen hacer "el camino de vuelta" (Betti, 1994) a la Educación Física, lo que genera una seria desconexión entre la producción de conocimiento y la especificidad del campo de la Educación Física ${ }^{5}$. En ese estado de cosas, la formación superior en la disciplina se presenta como escindida nuevamente entre teóricos y prácticos, pero esta vez entre teóricos que pretenden ser reconocidos como "científicos" de la Educación Física (PICH, S.; 2014: 18).
\end{abstract}

Siguiendo a Pich en la distinción entre teóricos y prácticos, es preciso remarcar que, en los institutos de formación docente terciaria no universitaria, los "teóricos", en general, no producen conocimiento científico

\footnotetext{
5 Aquí operamos con la noción de "campo de la Educación Física" desarrollada por la profesora Fernanda Paiva. Para esta autora, "a noção de campo indica um espaço social em que está em jogo a legitimidade de uma especificidade" (Paiva, 2004: 65). Más adelante volveremos sobre este punto.
} 
dado que la principal tarea es la docencia. Es decir, no cuentan con horas rentadas para la investigación. Sin embargo, es destacable la mención al área de investigación del campo en el diseño curricular que subraya la histórica vinculación entre las teorías de la Educación Física y el discurso de la Ciencia (positivista).

\section{3 - Escenario entorno a la continuidad pedagógica:}

Dado que la investigación se realizó durante el ciclo lectivo de 2020 en donde se suspendió la presencialidad y se dio continuidad a la enseñanza a partir del uso de herramientas de Educación a distancia, esta cuestión de la histórica distinción entre "teóricos" y "prácticos" se vio al menos observada. Decretada la emergencia sanitaria a partir de la pandemia producto del COVID 19, en Argentina se establece el DNU N²60/2020 mediante el cual se dicta la EMERGENCIA SANITARIA en todo el territorio nacional.

ACTUACIÓN DEL MINISTERIO DE EDUCACIÓN EN LA EMERGENCIA SANITARIA: EI MINISTERIO DE EDUCACIÓN establecerá las condiciones en que se desarrollará la escolaridad respecto de los establecimientos públicos y privados de todos los niveles durante la emergencia, de conformidad con las recomendaciones de la autoridad sanitaria, y en coordinación con las autoridades competentes de las distintas jurisdicciones (ARTíCULO 13) DNU 260-2020.

Luego que el Ministerio de Educación de la Nación decretó la emergencia sanitaria en todo el país, la provincia de Buenos Aires declaró la necesidad de dar continuidad pedagógica con herramientas de educación a distancia y suspensión de la presencialidad.

Es menester hacer alusión aquí al artículo 6 de la resolución 103/ 2020 del Ministerio de Educación de la Nación, con fecha del 12 de marzo del corriente año, donde se le solicita a las provincias y a la ciudad Autónoma de Buenos Aires, como así también a las Universidades, que -en el marco de sus competencias-, garanticen el derecho a la educación, disponiendo los dispositivos que consideren pertinentes (RODRÍGUEZ, N.; TRYBALSKI, M.: 2020:7).

Suspendida la presencialidad y garantizada la continuidad de la enseñanza y transmisión en todos los niveles y modalidades, la Formación 
Superior -de la que depende el Profesorado objeto de esta investigacióngarantizó continuar los procesos de formación y acreditación.

Modificados los procesos y frente a nuevos desafíos profesionales, las preguntas que surgen son: ¿qué saberes utilizan los docentes de enseñanza terciaria - no universitaria?, ¿qué vinculaciones se establecen entre los saberes docentes y los campos de investigación?, ¿qué objetivos, tipos de formulación, conceptos, elecciones teóricas operan en la enseñanza y transmisión en la formación de profesores?, ¿̇qué nuevos lugares operan en esta modalidad de educación en situación de ASPO?, ¿qué rol ocupan hoy las herramientas de Educación a distancia?

\section{METODOLOGÍA}

El presente trabajo se incluye dentro de los estudios cualitativos, habiéndose empleado técnicas de recolección de datos acordes: entrevistas y revisión y análisis de documentos. El estudio parte de la pregunta sobre el saber, para luego, dar lugar al análisis del diseño curricular del nivel superior en específico. Dicho análisis se focalizó principalmente en el estudio de los contenidos de las asignaturas, teniendo en cuenta que el Profesorado de Educación Física del Instituto Superior de Formación Docente Número 84 es una carrera terciaria no universitaria. Seguido a ello, se investigaron las leyes y conjunto de resoluciones y disposiciones que permitieron la continuidad pedagógica durante el año 2020 en situación de aislamiento social, preventivo y obligatorio (ASPO).

El diseño del instrumento (entrevista) contempló cuestionarios que fueron aplicados en profesoras y profesores de Educación Física que se desempeñan en su ejercicio profesional en el Instituto Superior de Formación Docente $\mathrm{N}^{\circ} 84$ de la ciudad de Mar del Plata de la provincia de Buenos Aires de la República Argentina. Centrando la pauta de la entrevista en la cuestión de la enseñanza de la teoría y la práctica.

Los datos fueron analizados, elaborándose categorías analíticas que fueron trianguladas con las fuentes recopiladas. Dicha triangulación permitió 
presentar un análisis de situación en la enseñanza y transmisión en dicha institución.

\section{PROCEDIMIENTO DE ANALISIS:}

El trabajo implicó un estudio sobre las nuevas condiciones del ASPO y la continuidad pedagógica, tomando como insumos las legislaciones nacionales y provinciales, la revisión y análisis del currículum de la Formación de Profesores/as en Educación Física de la provincia de Buenos Aires. A su vez, se realizó un trabajo de campo que consistió en entrevistas al cuerpo docente del Instituto Superior de Formación Docente Nro 84, situado en la ciudad de Mar del Plata, Buenos Aires, Argentina, con el propósito de desarrollar un análisis cualitativo sobre los procesos de enseñanza y transmisión en la Formación inicial de Profesores de Educación Física. El objetivo fue analizar los saberes que incumben en las prácticas profesionales. Si bien sabemos que la pregunta por el saber es compleja, el recorte temático se centró en las vinculaciones entre teoría y práctica en la formación de Profesores de Educación Física, durante el ciclo lectivo 2020, momento en que se suspendió la presencialidad en la enseñanza.

\footnotetext{
La suspensión de la presencialidad alteró la práctica profesional de, en este caso, las y los profesores de las Universidades. Todo ese saber del que se disponía para operar hasta ahora, se debió reconfigurar, resignificar, modificar para enseñar en este contexto. La nueva forma de enseñar que esta situación presentó requirió transformar las lógicas de la presencialidad. "Dar la clase" en esta circunstancia implicó dejar de lado "un saber docente" para aprender otro saber "dar la clase". Esto, porque, al alterarse la práctica profesional por la suspensión de la presencialidad, se requirió el involucramiento con "otros" saberes específicos de saber y transmitir (DALCEGGIO, M.; RODRÍGUEZ, N.; 2021:5).
}

Siguiendo con la entrevista, el cuestionario consistió en la participación de 11 personas 6 , mediante la herramienta Google Forms, lo que permitió indagar acerca de los discursos presentes sobre la relación entre teoría y práctica, de manera virtual, siguiendo los protocolos vigentes de sanidad.

\footnotetext{
"A modo de análisis, se menciona a cada persona como "caso" (enumerándolos de 111), de modo que permita extraer citas de su discurso sin comprometer su identidad.
} 
A partir de las respuestas, se analizaron recurrencias y discontinuidades en los discursos que permitieron concluir en cuatro categorías analíticas, que son las siguientes: 1. Relación entre teoría y práctica. 2. Jerarquización de los contenidos. 3. Interacción profesional vs. tecnología. 4. Estatus particular que los profesores le confieren a los saberes experienciales.

1. La relación entre teoría y práctica: tres de los once casos describieron su asignatura como práctica o teórica (caso 1-5-7). Mientras que los casos 2, 8, 10 y 11 consideran a la teoría y práctica como indivisibles y en tercer lugar, los casos 9 y 10 destacan en sus discursos la lejanía entre ambos términos.

Caso 8: "Ambos presentes. Uno sustenta el otro. Considero que la práctica se sustenta o fundamenta en la teoría y viceversa" Caso 10: "Ambas" Caso 11: "Práctico y Teórico, las dos cosas."

Los casos 3, 4, 9 también consideran a esta dupla como indivisible, pero sostienen que la teoría sustenta a la práctica.

Caso 2: "En ese ordenamiento lógico, la teoría siempre está primera." Caso 3: Pondera un orden teórico que se constituye en la práctica. Caso 4: "La considero un hacer fundado" "Cada forma de hacer remite a un marco teórico determinado." Caso 9: "Ponderan por igual, aunque el fin último sea mejorar la práctica propiamente dicha."

Y, los casos 9 y 10 destacan en sus discursos la lejanía entre ambos términos:

Caso 9: "La relación por momentos me parece adecuada, y por momentos inexistente, con teorías que se encuentran muy lejanas a la práctica, o con prácticas de las cuáles no existe teoría." Caso 10: "Muchas veces alejada la teoría de la realidad en la que nos enfrentamos en las escuelas o espacios de acción."

2. Jerarquización de los contenidos: tres de los once casos sostuvieron en sus discursos que la virtualidad en contexto de pandemia exigió una selección de contenidos (a su juicio) más importantes, ante la imposibilidad de trabajar todos los contenidos prescriptos en el diseño. 
Condicionados por problemas de conexión, sumado al desconocimiento (parcial o total) del uso de herramientas digitales, se produjo un enlentecimiento en la organización y cumplimiento de las clases.

Caso 2: "Hemos podido abordar todos los contenidos, quizás no con la profundidad habitual." Caso 5: "No. Actualmente intento dar por lo menos los más importantes". Caso 6: "Se seleccionaron contenidos."

3. Interacción profesional vs. tecnología. En principio, se evidencia una importante conmoción producto al gran esfuerzo que provocó la adaptación de las clases. Y en este apartado, no sólo entran en juego las aptitudes del equipo docente en dicha adaptación, sino cuestiones institucionales, económicas, culturales y políticas que florecen en este tipo de situaciones extremas mundiales como lo es la pandemia.

Priorizando la idea del saber y la vinculación entre la teoría y la práctica, queda de manifiesto que algunos profesores sostienen que la virtualidad imposibilita ciertos procesos en la dinámica de la enseñanza. Así queda expreso en los casos 2, 10, 4, 9.

Caso 2: "Afectó cierta dinámica de los encuentros presenciales, sobre todo aquellos momentos de debate y discusión." Caso 10: "Trabajar en la virtualidad no permitió (o no supe generarlo) un espacio real de intercambio." Caso 4: "Se complica mantener encuadres grupales para el intercambio." Caso 9: "La participación de los estudiantes es mucho menor, no sólo en entregas, sino también cuando no quieren prender sus cámaras y micrófonos durante un debate y sólo son espectadores de los pocos que sí lo hacen."

Entretanto, los casos 1 y 3, destacan de la virtualidad el uso de herramientas que permiten una continuidad interesante en la enseñanza.

Caso 1: "Las clases teóricas no sufrieron ninguna modificación más que en su forma (virtual). Los saberes se desarrollan y desarrollaron en plenitud."

Caso 3: "Las clases se dan con una interesante continuidad. Se profundizaron aspectos vinculados a las lecturas y reflexiones sobre las ideas."

A su vez, esta situación de virtualidad deja en evidencia el déficit que existe en el estudio y desarrollo de competencias digitales o virtuales durante la Formación Docente. 
Caso 5: "Los conocimientos tecnológicos al inicio fueron insuficientes." Caso 6: "Organizar clases virtuales sin estar previstas. Los docentes en su mayoría desconocíamos estas herramientas que luego poco a poco se fueron implementando." Caso 8: "Usamos por primera vez, aprendimos su uso y aplicamos a nuestro campo, diferentes recursos digitales." Caso 10: "Aprendizaje por medio de tutoriales (falta de capacitación en el uso de las tics)"

Pero, todos los casos coinciden con que esto provocó un cambio muy grande en la manera de enseñar. Transformación que perdurará (o al menos eso se espera), en torno al uso de la virtualidad y la tecnología como herramientas educacionales, que antes no eran tenidas en cuenta.

Caso 1: "Ayudará a saber o a tener más conocimiento de herramientas digitales para amplificar las posibilidades de acceso al saber." Caso 2: "Seguir vinculando a cierta cultura digital". Caso 3: "Va a continuar el ámbito virtual como medio de soporte y complementos a los escenarios presenciales". Caso 4: "El uso de herramientas digitales". Caso 7: "Entiendo que los recursos digitales pueden ser un excelente complemento de la tarea docente en formato presencial". Caso 9: "Las clases prácticas como eran antes, y el material visual como apoyo, puede ser de gran ayuda".

4. Estatus particular que los profesores le confieren a los saberes experienciales: tres de los once casos expresan la importancia de la experiencia en la Formación Docente. Incluye el aprendizaje, principalmente de saberes relativos a las situaciones que emergen día a día en el aula, en interacción con el alumnado y los diferentes actores.

Caso 1: "Nos encontramos con la dificultad de poder sintetizar los contenidos en la práctica." Caso 4: "En cuanto a las dificultades, se complica mantener encuadres grupales para el intercambio. Y el despliegue en terreno de los aspectos instrumentales." Caso 9: "La mayor dificultad es el hecho de vivir la experiencia de la práctica, sentir en carne propia cuáles son las dificultades de ambos deportes en sus diferentes aspectos, para que el día que me toque llevarlos a la práctica como docente, poder reconocer esas dificultades en el otro."

En el apartado siguiente se presenta un análisis y discusión sobre las cuatro categorías descriptas anteriormente, surgidas del trabajo de campo. 


\section{RESULTADOS}

Dadas las categorías sociales y analíticas resultantes que se enuncian en esta investigación, surge la importancia de conceptualizar los problemas de la teoría y las prácticas en la enseñanza y transmisión, implicadas en la formación inicial de profesores en Educación Física.

Archenti (2017) señala que la vinculación entre la teoría y los datos es relevante y así, la particular relación entre teoría, método y datos le otorga a cada investigación un carácter específico.

"La teoría contiene tres elementos fundamentales que constituyen el marco o el horizonte para el desarrollo metodológico:

a) Las categorías: que operan como códigos interpretativos de la realidad y orientan la mirada hacia determinados aspectos y problemas. (...)

b) Los criterios de relevancia: que establecen los límites del universo de problemas y de datos, dando respuestas a la pregunta żcuáles son los problemas que atender?

c) El registro de los límites del conocimiento científico en cada momento histórico, que fijan el punto de partida de la mirada y el horizonte cognitivo para la producción de nuevas teorías con mayor poder heurístico." (ARCHENTI, N.; 2017: 66).

Así, las funciones que las teorías cumplen dentro de la investigación son diversas y existe una intrínseca relación entre teorías, investigación y producción de conocimiento. Entendemos a las prácticas a la manera de Foucault, como modos de hacer, pensar y decir y en este marco, presentamos una perspectiva crítica frente a las teorizaciones que reducen el campo de la Educación Física, a teorías del movimiento o de la corporeidad humana. ¿Por qué?, ¿cuáles son los límites de estas perspectivas?

En correlato con lo antedicho, acordamos con Archenti (2007) cuando afirma que conceptualizar el término "teoría" es muy complejo, dado que definir la teoría sólo es posible en el marco de un sistema teórico. Si bien hay bastante consenso en suponer que una teoría reúne un conjunto de conceptos que se relacionan entre sí, la autora afirma que Merton es quien, tal vez, conceptualice mejor esta idea, citando:

"se dice a veces que la teoría está formada por conceptos, afirmación que por ser incompleta no es verdadera ni falsa, sino vaga [...] Sólo cuando tales conceptos se 
relacionan entre sí en forma de un sistema, empieza a aparecer la teoría" (MERTON 1949/1964:99 EN ARCHENTI 2007: 62).

Si bien es compleja la relación entre teorías y prácticas y su correlación no es lineal, lo cierto es que las y los docentes que participaron de la investigación a través de las entrevistas, se enuncian al menos tres posiciones diferenciadas. Es decir, unos expusieron su asignatura como práctica o teórica, otros consideraron a la teoría y práctica como indivisibles y otro grupo destacó la lejanía entre ambos términos.

Por su parte, Tardif, M. (2007), a partir de analizar los saberes de los docentes, problematiza la compleja vinculación entre los saberes disciplinares y el conjunto de saberes necesarios para el desarrollo de las prácticas profesionales. Este punto de partida, permite al autor "repensar las concepciones tradicionales referentes a la relación entre teoría y práctica y en especial, las relaciones entre la investigación universitaria y la práctica del oficio de docente." (TARDIF, M; 2007; 168).

El caso de la Educación Física no es aislado, si bien las investigaciones en el campo se producen mayormente en las Universidades y en los centros especializados de investigación -sean éstos públicos o privados-, la formación de grado en Universidades permite la iniciación en investigación a partir de elaboraciones de tesis de licenciatura, al igual que en posgrado. De esta manera, los Institutos de Formación Superior -como el estudiado- no son necesariamente centros de investigación científica y por lo tanto, las investigaciones se realizan de forma aislada. Dicha cuestión intensifica, aún más, la histórica y compleja vinculación entre teoría y práctica, siendo las clases producto de una enseñanza de teorías realizadas por otros.

Con este enfoque, un docente no es sólo aquel que reproduce conocimientos producidos por otros, sino un actor que asume su práctica "a partir de los significados que él mismo le da, un sujeto que posee conocimientos y un saber hacer provenientes de su propia actividad y a partir de los cuales la estructura y la orienta." (TARDIF, M; 2007; 169) Esto es, situando al docente como sujeto competente y activo (como sujeto de conocimiento) y a la práctica considerándola no sólo como un espacio de 
aplicación del saber, sino más aún como un espacio de producción y transformación. Es decir, una práctica por sí misma provista de saber.

Así, las prácticas no se piensan de manera aislada a la teoría, sino como una relación de relevos, entre la teoría, las acciones sobre los otros (poder) y en la relación a cómo nosotros mismos pensamos el saber y el poder (subjetividad). La práctica, en este contexto, no es "ciega" (como sí podríamos considerar a la técnica, en la medida que no producen la teoría que aplican). Por el contrario, las prácticas tienen la característica de reflexionar sobre sí mismas. Si asumimos que practica no es sólo un espacio de aplicación de saberes provenientes de la teoría, sino también un sistema donde se producen saberes que provienen de esa misma práctica, el docente ya no es aquel que reproduce saberes creados por otros, sino un sujeto de conocimiento, actor que desarrolla y posee teorías y saberes de su propia acción. Es decir, ambas, teoría y práctica, "[..] son portadoras y productoras de prácticas y de saberes, de teorías y acciones y ambas comprometen a los actores, sus conocimientos y sus subjetividades" (TARDIF, M; 2007;174)

Por su parte, respecto al problema de la jerarquización de los contenidos -que ya ha sido estudiado en otras investigaciones- (Rodríguez, N. B.; 2015-2017; Rodríguez, N.B.; Fernandez Vaz, A.2016), se puede visualizar que en su proceso de organización para la enseñanza, se seleccionan y jerarquizan los saberes a enseñar. Sin embargo, en este período estudiando, las y los profesores manifiestan haber tenido que reorganizar o volver a realizar estos procesos, dada la cuestión de la continuidad de la enseñanza mediada por herramientas de educación a distancia.

Asimismo, en el tema de interacción profesional vs. tecnología se registra que los y las docentes manifiestan un desconocimiento inicial frente a los desafíos tecnológicos que, a su vez, ponen en tensión el estatus particular que los profesores le confieren a los saberes experienciales. Las tradiciones respecto de la enseñanza "práctica" y el valor otorgado a la experiencia en tanto vivencia, dejan de manifiesto que en el área no sólo existen tensiones entre teoría y práctica como en otras áreas de 
conocimiento sino que, a su vez, la ponderación de la práctica sobre la teoría vislumbra la incertidumbre frente a una enseñanza en la que el cuerpo físico se ausenta. Medida por pantallas, la enseñanza de la Educación Física "práctica" requirió un conjunto de competencias diferenciadas y una necesaria revisión de los saberes de la enseñanza.

\section{CONCLUSIONES}

En función del estudio realizado, es relevante destacar y retomar el conjunto de categorías analíticas que se elaboraron: relación entre teoría y práctica; jerarquización de los contenidos; interacción presencial vs tecnología y sobre el estatus particular que los profesores le confieren a los saberes experienciales. Cómo se desarrolló en el apartado anterior no es posible abordar una sin las otras. Las categorías forman parte de un marco teórico que, indefectiblemente, puede ser analizado en tanto la necesaria implicación de unas con otras. El tema histórico de las tensiones entre teoría y práctica, se vio de manifiesto en la enseñanza mediada por herramientas de educación a distancia siendo, a su vez, la cuestión de la jerarquización de contenidos un tema puesto de manifiesto. Asimismo, ello se vio interpelado por la sobrevaloración que existe en la Formación de profesores y profesoras de Educación Física respecto a las "enseñanzas prácticas vinculadas con situaciones experienciales". Si bien no es objeto de este artículo, lo cierto es que, para enseñar la enseñanza del vóley, por ejemplo, se hacen prácticas y entrenamientos de vóley. Es decir, hay una primacía de teorías que afirman la necesidad de realizar la experiencia práctica para luego poder transmitir ese saber. Como señalamos en otras oportunidades, lo cierto es que esta emergencia sanitaria puso de manifiesto problemas históricos del campo que precisan ser atendidos.

\section{REFERENCIAS}

ARCHENTI, N.; (2017) El papel de la teoría en la investigación social. Marradi, A., Archenti, N., Piovani, J. (2007) Metodología de las Ciencias Sociales. Emecé Editores. Buenos Aires. 
CRISORIO, R.; ESCUDERO, C, coordinadores (2017). Educación del cuerpo: Currículum, sujeto y saber. La Plata: Universidad Nacional de La Plata. Facultad de Humanidades y Ciencias de la Educación. (Cuerpo, educación y sociedad; 1). En Memoria Académica. Disponible en: http://www.memoria.fahce.unlp.edu.ar/libros/pm.504/pm.504.pdf

DALCEGGIO, M. M., \& RODRíGUEZ, N. B. (2021). Theory and practice in teacher training at UNLP: an analysis of the curriculum. Journal of Research and Knowledge Spreading, 2(1), e12303. https://doi.org/10.20952/jrks21 12303

DE ALBA, A. (2007). Crisis, mito y perspectiva. España: Ediciones Miño y Dávila.

GILES, M. 2017. Prácticas Corporales En: Crisorio, R.; Escudero, C, coordinadores. Educación del cuerpo: Currículum, sujeto y saber. La Plata: Universidad Nacional de La Plata. Facultad de Humanidades y Ciencias de la Educación.

GOMEZ, R, H (2017) La investigación de la enseñanza y el aprendizaje en educación física: núcleos problemáticos en la perspectiva de la didáctica comparada En libro: Crisorio, R. L. y Escudero, C. (coord.). Educación del cuerpo: Currículum, sujeto y saber. La Plata: Universidad Nacional de La Plata. Facultad de Humanidades y Ciencias de la Educación.

GVIRTZ, SILVANA Y MARIANO PALAMIDESSI (1998). "La construcción social del contenido a enseñar", en El $A B C$ de la tarea docente: Currículo y enseñanza. Buenos Aires: Aiqué, pp. 17-48.

PICH S.; La formación de formadores y de profesores de Educación Física en Brasil: la tensión entre la producción de conocimiento y la identidad epistemológica, En: E. Camblor, O. Ron, N. Hernández, G. Fittipaldi, M. Uro y A. Mele (Coords.). Prácticas de la educación física. La Plata: Universidad Nacional de La Plata. Facultad de Humanidades y Ciencias de la Educación. (Colectiva y monográfica; 2 ) Disponible en: http://www.memoria.fahce.unlp.edu.ar/libros/pm.393/pm.393.pdf

RODRÍGUEZ, NORMA BEATRIZ, directora. (2020) Producción Científica y Formación de Educadores Corporales. Estudio sobre la Formación inicial de profesores en Educación Física en la UNLP / H951. (Proyecto de investigación). UNLP. FaHCE. Centro Interdisciplinario Cuerpo, Educación y Sociedad. Disponible en:

ttp://www.memoria.fahce.unlp.edu.ar/proyectos/py.995/py.995.pdf

RODRÍGUEZ, N. (2015). LOS contenidos de la educación física. EN: R. Crisorio y M. Giles (Dirs.). Estudios críticos de educación física. La Plata: Al Margen.

RODRÍGUEZ, N.; FERNANDEZ VAZ, A.(2016) Contenidos de la enseñanza: Conceptualizaciones, crítica epistemológica, propuestas de intervención. 
Didaskomai, 7: 44-56. Disponible en:

http://didaskomai.fhuce.edu.uy/index.php/didaskomai/article/view/22

RODRÍGUEZ, N. (2017). Contenidos de la educación física. Aportes para analizar la enseñanza y la transmisión. EN: S. Achucarro, N. Hernández y D. Di Domizio (Comps.). Educación física: Teorías y prácticas para los procesos de inclusión. La Plata: Universidad Nacional de La Plata. Facultad de Humanidades y Ciencias de la Educación.

RODRÍGUEZ, N.; TRYBALSKI, M. (2020) Plan de continuidad pedagógica, educación física y estado de excepción: Estudio sobre la educación secundaria en la provincia de Buenos Aires. Tempos e espaços em educação, 13 (32): el 4088. Disponible en:

http://www.memoria.fahce.unlp.edu.ar/art_revistas/pr.1 1873/pr.1 1873.pdf

TARDIF, M. (2004). Los saberes del docente y su desarrollo profesional. Madrid: Narcea.

VALLINA, J.; RODRÍGUEZ, N. (2016). Debates curriculares actuales. La educación física en cuestión. 11 Congreso Argentino de Educación Física y Ciencias, 28 de septiembre al 2 de octubre de 2015, Ensenada, Argentina. En: Publicaciones 11 Congreso (2015). Ensenada: Universidad Nacional de La Plata. Facultad de Humanidades y Ciencias de la Educación. Departamento de Educación Física. Disponible en:

http://www.memoria.fahce.unlp.edu.ar/trab_eventos/ev.7240/ev.7240.pdf

\section{Documentos y fuentes consultadas:}

DISEÑO CURRICULAR DE LA PROVINCIA DE BUENOS AIRES. Dirección General de Cultura y Educación Provincia de Buenos Aires Año 2009.

http://servicios2.abc.gov.ar/lainstitucion/organismos/consejogeneral/disenios curriculares/documentosdescarga/res2432-09fd_educ_fisica.pdf

LEY DE EDUCACIÓN NACIONAL (Ley 26206/06). Disponible en: https://www.argentina.gob.ar/sites/default/files/ley-de-educ-nac58ac89392ea4c.pdf

REPÚBLICA ARGENTINA. PRESIDENCIA. Decreto de Necesidad y Urgencia 260/2020. "Ampliación de la emergencia pública en materia sanitaria establecida en virtud de la pandemia en relación con el coronavirus COVID19". (DECNU-2020-260-APN-PTE.). Boletín Oficial de la República Argentina en 12/3/2020. Disponible en:

https://www.boletinoficial.gob.ar/suplementos/2020031201NS.pdf.

Consultada el 8/6/2020 
REPÚBLICA ARGENTINA. PRESIDENCIA. Decreto de Necesidad y Urgencia 297/2020. "Disposición del aislamiento social, preventivo y obligatorio del 20/3/20 al 31/3/20". (DECNU-2020-297-APN-PTE.).

BOLETÍN OFICIAL DE LA REPÚBLICA ARGENTINA el 19/3/2020. Disponible en: https://www.boletinoficial.gob.ar/detalleAviso/primera/227042/20200320. Consultada el 8/6/2020

REPÚBLICA ARGENTINA. MINISTERIO DE EDUCACIÓN DE LA NACIÓN Resolución 106/2020 (RESOL-2020-106-APN-ME). Boletín Oficial de la República Argentina, 15/03/2020. Disponible en:

https://www.boletinoficial.gob.ar/detalleAviso/primera/226751/20200316. Consultada el: 8/6/2020

Recebido em: 11 de maio de 2021 Aprovado em: 03 de julho de 2021

Publicado em: 15 de julho de 2021. 